\title{
Estimation de l'apport en mercure à partir de la consommation de poisson en Côte d'Ivoire
}

\author{
Kouamé M. KoffI ${ }^{12}$, Godi H. M. BIEGo ${ }^{1 *}$, Yolande AKE-AssI ${ }^{2}$ \& N'Zi G. Agbo'. \\ ${ }^{1}$ Laboratoire de Biochimie et Sciences des Aliments, UFR Biosciences, Université de Cocody, 22 BP 582 Abidjan 22, Côte d'Ivoire ou \\ 25 BP 313 Abidjan 25, Côte d'Ivoire \\ ${ }^{2}$ Laboratoire Central pour l'Hygiène Alimentaire et l'Agro-industrie, LANADA, Ministère de la production animale et des ressources \\ halieutiques, 04 BP 612 Abidjan 04, Côte d'Ivoire. \\ *Auteur pour les correcpondances (E-mail : biegoh3@yahoo.fr) \\ Reçu le 27-04-2006, accepté le 01-12-2006.
}

\begin{abstract}
Résumé
L'objectif de cette étude est d'estimer l'apport en mercure à partir des concentrations en cet élément de différentes espèces de poissons les plus consommées et les plus vendues en Côte d'Ivoire. Pour ce faire, 18 espèces de poissons couramment consommées et quelques prédateurs ont été échantillonnées selon la méthode du panier de la ménagère, dans des stocks de production locale et d'importation de 1999 à 2002. Les échantillons, après minéralisation par voie humide, ont été analysés par Spectrophotométrie d'Absorption Atomique selon la technique des vapeurs froides. Les concentrations en mercure retrouvées varient entre 8 et $2454 \mu \mathrm{g} / \mathrm{kg}$ avec une moyenne de $239 \mu \mathrm{g} / \mathrm{kg}$. Sur la base des concentrations et de la consommation annuelle de poissons estimée à $16 \mathrm{~kg} / \mathrm{habitant}$ en Côte d'Ivoire, l'apport moyen calculé est égal à $73 \mu \mathrm{g}$ de mercure par semaine; soit $25 \%$ de la dose hebdomadaire tolérable provisoire (DHTP) fixée à $300 \mu \mathrm{g}$ par le comité mixte $\mathrm{FAO} / \mathrm{OMS}$. Cependant, pour une population lagunaire, consommant essentiellement des poissons (consommation quotidienne de $1,08 \mathrm{~kg}$ selon l'OMS), le risque apparaît beaucoup plus élevé car l'apport en mercure est égal à $1703 \mu \mathrm{g} / \mathrm{semaine}$; soit 7 fois la DHTP.
\end{abstract}

Mots clés : apport, mercure, consommation, poissons, Côte d'Ivoire

\section{Abstract}

Assessment of dietary mercury intake from fish Consumption in Côte d'lvoire

The aim of this study is to determine mercury content in different species of fishes in order to estimate the daily mercury intake in adult Ivorian. Eighteen fish species were sampled in stocks. of Ivorian fish production and importation during four years (1999-2002). The mercury concentrations were determined by Atomic Absorption Spectrophotometer according to the cold vapours method.

The mercury average level is $239 \pm 196 \mu \mathrm{g} / \mathrm{kg}(2-2454 \mu \mathrm{g} / \mathrm{kg})$ in fishes. The intake value estimated is $73 \mu \mathrm{g}$ per week. This value represents $25 \%$ of Provisional Tolerable Weekly Intake recommended by the World Health Organization (300 $\mu \mathrm{g}$ per week). However, for an inhabitant in costs, the risk appears much higher because the intake value estimated is $1703 \mu \mathrm{g}$ per week; which represents 7 times the Provisional Tolerable Weekly Intake.

Key words : mercury, intake, fish, Côte d'lvoire 


\section{Introduction}

Le mercure est un élément toxique qui n'exerce aucune fonction physiologique utile chez I'homme (Schwarz, 1977; Bennett, 1984). La manifestation, le caractère et l'importance des effets dépendent de la forme chimique du métal, la nature du composé mercuriel, la quantité absorbée, la durée de l'exposition et la voie d'administration (Berlin, 1986). Les composés mercuriels inorganiques ont pour principale cible les reins où ils s'accumulent sous forme de métallothionéine. Les dérivés organiques, en particulier le méthylmercure (forme la plus toxique), sont neurotoxiques, néphrotoxiques, mutagènes, cancérogènes et tératogènes (OMS, 1980; Granjean et al., 1998; Crump et al., 1998).

La biométhylation du mercure est mise en évidence dans les sédiments et les organismes aquatiques (poissons, crustacés et coquillages). Ainsi, le méthylmercure se retrouve chez les poissons après concentration par le phytoplancton et le zooplancton (Granjean et al. 1995). Les quantités de méthylmercure peuvent varier selon l'espèce, l'âge et la taille des poissons; et les concentrations retrouvées peuvent atteindre $10 \mu \mathrm{g} / \mathrm{kg}$ (OMS, 1996). Par conséquent, la consommation de poissons peut être considérée comme l'une des principales sources d'apport en cet élément chimique et ces dérivés alkylés chez l'homme (Huss, 1996).

Compte tenu du caractère cumulatif du mercure et de ses dérivés alkylés, un contrôle régulier du contenu en mercure des différentes espèces de poissons consommées s'impose en Côte d'Ivoire. En dehors des données de Métongo et Kouamenan (1991), il n'existe pas de publications sur le niveau de contamination des produits de pêche dans notre pays. Aussi, notre étude se propose-t-elle d'estimer l'apport en mercure lié à la consommation de poissons en Côte d'Ivoire.

\section{Matériel et méthodes}

\subsection{Echantillonnage}

Les échantillons ont été prélevés sur 18 espèces de poissons, de consommation courante ou ayant une valeur marchande dans des stocks de production locale et d'importation de 1999 à 2002.
II s'agit de 192 échantillons de Carpes (Pomadasyidae; Pomadasys sp. et Plectorbiuchus mediterraneus), 144 de sardines (Clupeidae; Sardinella sp.), 192 de dorades (Sparidae; Dentex sp. et Pagrus sp.), 192 de soles (Soleidae; Cynoglossus sp.), 192 de mérous (Serranidae; Epinephelus sp.), 192 de capitaines (Polynemidae; Polydactylus sp.), 192 d'ombrines (Scianenidae; Pseudotolithus sp.), 192 de mâchoirons (Ariidea), 96 de pageots (Sparidae; Pagellus sp.), 96 de maquereaux (Scombridae; Scomber japonicus), 96 de chinchards (Carangidae; Trachurus trachurus et Decapterus sanctaelenae), 1021 de thons (Thonnidae; Katsuwonus pelamis et Thunnus sp.), 48 de voiliers (Istiophoridae; Istiophorus albicans), 57 d'espadons (Xiphiidae; Xiphias gladius), 53 de requins (Carcharitinus sp., Prinace $s p$. et Rhiniodon sp.), 48 de marlins (Makiadidae; Makaira nigricans), 48 de coryphènes (Coryphenidae; Coryphaena hippurus) et 48 de barracudas (Sphyraenidae; Sphyraena sp.); soit en tout 3099 échantillons.

\subsection{Minéralisation et analyse}

\subsubsection{Réactifs}

Les réactifs utilisés sont l'acide chlorhydrique fumant 37\% (Merck), l'acide nitrique 65\% (Merck), l'acide perchlorique $60 \%$ (Merck), l'acide sulfurique $96 \%$ (Merck), le chlorure d'étain (II) (Merck), le standard de mercure (Merck) et l'eau UHQ à $18 \mathrm{MW}^{-1}$.

\subsubsection{Minéralisation}

Un aliquote de $1 \mathrm{~g}$ d'homogénéisât de chaque poisson a été minéralisé à chaud en présence de $1 \mathrm{ml}$ du mélange sulfonitrique-perchlorique ( $5 \mathrm{ml}$ d'acide nitrique, $10 \mathrm{ml}$ d'acide sulfurique et $2 \mathrm{ml}$ d'acide perchlorique) à $250^{\circ} \mathrm{C}$ pendant 30 minutes. Le minéralisât est réduit par une solution de chlorure d'étain (II) à $10 \%$. La solution obtenue est conservée au réfrigérateur avant dosage.

\subsubsection{Appareillage et conditions de détermination du mercure}

Un spectrophotomètre d'absorption atomique (Shimadzu AA 660 muni d'une unité de vaporisation MUV) a été utilisé pour le dosage du mercure. La détermination du mercure a été 
réalisée à la longueur d'onde de 253,7 nm. Les concentrations en mercure ont été calculées grâce à un étalonnage externe établi en 6 points $(0,2 \mathrm{mg} / \mathrm{kg}, 0,4 \mathrm{mg} / \mathrm{kg}, 0,6 \mathrm{mg} / \mathrm{kg}, 0,8 \mathrm{mg} / \mathrm{kg}, 1$ $\mathrm{mg} / \mathrm{kg}$ et $1,5 \mathrm{mg} / \mathrm{kg}$ ). Les conditions opératoires du spectrophotomètre d'absorption atomique sont présentées dans le tableau 1.

Tableau 1 : Conditions opératoires du Spectro photomètre d'Absorption Atomique.

\begin{tabular}{lc}
\hline Désignation & Valeurs \\
\hline Intensité du courant & $4 \mathrm{~A}$ \\
Largeur de la fente & $0,5 \mathrm{~mm}$ \\
Longueur d'onde & $253,7 \mathrm{~nm}$ \\
Coefficient de variation & $1 \%$ \\
Temps d'intégration & 5 secondes \\
Nombre de répétitions & 3 \\
\hline
\end{tabular}

\subsection{Méthode de validation}

La validation des techniques d'analyse et de minéralisation a été réalisée selon la méthode de l'Association Française de Normalisation (AFNOR, 1996). Elle a consisté en la détermination des limites de détection et de quantification; au calcul du coefficient de variation du mercure pour les essais de répétabilité et de reproductibilité et au calcul du pourcentage de recouvrement de mercure ajouté avant et après minéralisation. La validation a été effectuée à l'aide de 2 standards de mercure (standard $A$ : $225 \mu \mathrm{g} / \mathrm{L}$; standard B: $600 \mu \mathrm{g} / \mathrm{L}$ ). A partir du témoin issu de la minéralisation, l'analyse de 30 essais distincts a permis de calculer les limites de détection et de quantification selon les formules suivantes:

Limite de détection $(L D)=M x+2 " 2 t_{0,95} s$

Limite de quantification $(L Q)=M x+10 s$

$M x$ : valeur d'information moyenne du témoin

$s$ : écart type des valeurs d'information sur le témoin

$2 " 2 t_{0,95} s$ : est égal à 4,8 pour un test unilatéral de Student's; $a=0,05$ et $n=30$

\subsection{Estimation de l'apport en mercure}

L'apport en mercure a été estimé à partir des données de la consommation de poisson de la Direction des Productions Halieutiques de Côte d'Ivoire (DPH) et celles du système de contrôle de l'environnement de l'Organisation Mondiale de la Santé. Les quantités de poissons consommées sont respectivement de 43,8 g/jour/ personne (DPH, 2002) et de 36,5 g/jour/personne (OMS, 2003) selon ces deux institutions. Par ailleurs, l'apport en mercure d'un sujet qui ne consomme essentiellement que du poisson a été estimé à partir de la consommation alimentaire quotidienne d'un africain adulte. Cette consommation est égale à 1018,1 $\mathrm{g}$ /jour selon l'OMS (OMS, 2003). L'apport en mercure a été calculé selon la formule suivante (OMS, 2003):

Apport en mercure $=T_{H g} \times C$

$T_{H g}$ : teneur en mercure retrouvée dans l'aliment $(\mu g / k g)$

$C$ : consommation alimentaire quotidienne de l'individu ( $\mathrm{kg} / \mathrm{jour})$

\section{Résutats et discussion}

\subsection{Résultats de la validation}

Les résultats de la validation sont présentés dans le tableau 2. Les limites de détection et de quantification sont respectivement $0,25 \mu \mathrm{g} / \mathrm{L}$ et $0,28 \mu \mathrm{g} / \mathrm{L}$. Les coefficients de variation sont compris entre $1,9 \%$ et $2,1 \%$ pour la répétabilité et entre $4,2 \%$ et $5,3 \%$ pour la reproductibilité. Le pourcentage de recouvrement des ajouts dosés sont, avant minéralisation, de $96,8 \%$ pour le standard A et de $97,5 \%$ pour le standard B. Après minéralisation, ces ajouts sont récupérés à $102,6 \%$ pour le standard $A$ et à $103,8 \%$ pour le standard $B$. Ces résultats mettent en évidence la fiabilité et la précision satisfaisante des techniques de minéralisation et d'analyse.

\subsection{Concentration en mercure des échantillons}

Les concentrations en mercure des différents échantillons analysés sont présentés dans le tableau 3. La concentration moyenne pondérée de tous les échantillons est de $239 \pm 196 \mu \mathrm{g} / \mathrm{kg}$ 
$(8-2454 \mu \mathrm{g} / \mathrm{kg})$. Les teneurs les plus élevées sont retrouvées au niveau des prédateurs: marlin $755 \pm$ $\mu \mathrm{g} / \mathrm{kg}(117-2454 \mu \mathrm{g} / \mathrm{kg})$, requin $705 \pm \mu \mathrm{g} / \mathrm{kg}(122-$ $1725 \mu \mathrm{g} / \mathrm{kg})$, espadon 699 $\pm \mu \mathrm{g} / \mathrm{kg}(24-2153 \mu \mathrm{g} /$ $\mathrm{kg}$ ) et voilier $555 \pm \mu \mathrm{g} / \mathrm{kg}(20-1191 \mu \mathrm{g} / \mathrm{kg})$. Ces teneurs peuvent s'expliquer par la position de ces poissons dans la chaîne alimentaire (Boisset, 1996). Par ailleurs, on note que certaines concentrations sont supérieures à la valeur maximale fixée pour les prédateurs $(1000 \mu \mathrm{g} / \mathrm{kg})$ (Boisset, 1996), ce qui pourrait constituer un risque pour le consommateur.

La concentration moyenne en mercure des poissons du stock local $(333 \pm 230 \mu \mathrm{g} / \mathrm{kg})$ est 2 fois celle des poissons du stock importé $(160 \pm 102$ $\mu \mathrm{g} / \mathrm{kg}$ ). Par ailleurs, le stock local se compose de $7,7 \%$ d'échantillons ayant une concentration comprise entre $500-1000 \mu \mathrm{g} / \mathrm{kg}$ et $1,2 \%$ une concentration supérieure à $1000 \mu \mathrm{g} / \mathrm{kg}$. Aucun échantillon du stock importé n'a une teneur en mercure supérieure à $1000 \mu \mathrm{g} / \mathrm{kg}$ (tableau 4). Seul le stock local contient des prédateurs.

Tableau 2 : résultats de la validation des techniques

\begin{tabular}{lc}
\hline Désignation & Valeur \\
\hline $\begin{array}{l}\text { Concentration moyenne } \\
(n=30)\end{array}$ & \\
Blanc & $0,21 \pm 0,01 \mu \mathrm{g} / \mathrm{L}$ \\
Standard A & $225 \pm 21 \mu \mathrm{g} / \mathrm{L}$ \\
Standard B & $600 \pm 48 \mu \mathrm{g} / \mathrm{L}$ \\
& \\
Coefficient de variation de la répétabilité & \\
$(n=10)$ & \\
Blanc & $1,9 \%$ \\
Standard A & $2,1 \%$ \\
Standard B & $2,0 \%$ \\
& \\
Coefficient de variation de la reproductibilité & \\
$(n=30)$ & \\
Blanc & \\
Standard A & $4,2 \%$ \\
Standard B & $5,3 \%$ \\
& $4,6 \%$ \\
Pourcentage de recouvrement des ajouts dosés & \\
$(n=5)$ & \\
Avant minéralisation & \\
Standard A & \\
Standard B & $96,8 \pm 5,7 \%$ \\
Après minéralisation & $97,5 \pm 6,3 \%$ \\
Standard A & \\
Standard B & $102,6 \pm 4,2 \%$ \\
Limite de détection & $103,8 \pm 5,3 \%$ \\
Limite de quantification & $0,25 \mu \mathrm{g} / \mathrm{L}$ \\
\hline
\end{tabular}


Tableau 3 : concentrations en mercure retrouvées dans les échantillons de poissons

\begin{tabular}{|c|c|c|c|}
\hline \multirow{2}{*}{ Espèces } & \multirow{2}{*}{$\mathbf{n}$} & \multirow{2}{*}{ Moyenne } & \multirow{2}{*}{$\frac{\text { Valeurs limites }}{\text { (Mini-Maxi) }}$} \\
\hline & & & \\
\hline \multicolumn{4}{|l|}{ Stock du littoral ivoirien } \\
\hline Carpes & 192 & $192 \pm 44$ & $72-309$ \\
\hline Sardines & 48 & $161 \pm 16$ & $129-220$ \\
\hline Capitaines & 192 & $178 \pm 39$ & $84-279$ \\
\hline Daurades & 192 & $158 \pm 42$ & $68-276$ \\
\hline Mâchoirons & 192 & $168 \pm 44$ & $71-296$ \\
\hline Soles & 192 & $109 \pm 30$ & $8-198$ \\
\hline Mérous & 192 & $175 \pm 44$ & $69-298$ \\
\hline Ombrines & 192 & $155 \pm 36$ & $67-276$ \\
\hline Thons & 1021 & $271 \pm 187$ & $9-1281$ \\
\hline Voiliers & 48 & $555 \pm 221$ & $20-1191$ \\
\hline Espadons & 57 & $699 \pm 352$ & $24-2153$ \\
\hline Requins & 53 & $705 \pm 359$ & $122-1725$ \\
\hline Marlins & 48 & $755 \pm 482$ & $117-2454$ \\
\hline Coryphènes & 48 & $373 \pm 165$ & $85-943$ \\
\hline Barracudas & 48 & $337 \pm 143$ & $93-710$ \\
\hline Moyenne stock du littoral & 2715 & $333 \pm 230$ & $8-2454$ \\
\hline \multicolumn{4}{|l|}{ Stock importé } \\
\hline Maquereaux & 96 & $148 \pm 75$ & $22-431$ \\
\hline Chinchards & 96 & $249 \pm 146$ & $17-630$ \\
\hline Pageots & 96 & $138 \pm 52$ & $38-267$ \\
\hline Sardines & 96 & $104 \pm 33$ & $48-234$ \\
\hline Moyenne stock importé & 384 & $160 \pm 102$ & $17-630$ \\
\hline MOYENNE TOTAL & 3099 & $239 \pm 196$ & $8-2454$ \\
\hline
\end{tabular}

Concentration en $\mu \mathrm{g} / \mathrm{kg}$ de poids frais

Tableau 4 : représentation du contenu en mercure des échantillons par rapport aux normes

\begin{tabular}{lcc}
\hline Contenu en mercure des échantillons & Nombre d'échantillons & Pourcentage \\
\hline Stock total & 3099 & 100 \\
$0,28-500 \mu \mathrm{\mu g} / \mathrm{kg}$ & 2814 & 90,8 \\
$500-1000 \mu \mathrm{g} / \mathrm{kg}$ & 248 & 8,0 \\
$>1000 \mu \mathrm{gg} / \mathrm{kg}$ & 47 & 1,9 \\
Stock $\mathrm{local}$ & 2715 & $\mathbf{8 7 , 6}$ \\
$0,28-500 \mu \mathrm{kg} / \mathrm{kg}$ & 2439 & 78,7 \\
$500-1000 \mu \mathrm{g} / \mathrm{kg}$ & 239 & 7,7 \\
$>1000 \mu \mathrm{g} / \mathrm{kg}$ & 37 & 1,2 \\
Stock importé & 384 & $\mathbf{1 2 , 4}$ \\
$0,28-500 \mu \mathrm{g} / \mathrm{kg}$ & 375 & 12,1 \\
$500-1000 \mu \mathrm{g} / \mathrm{kg}$ & 9 & 0,3 \\
$>1000 \mu \mathrm{gg} / \mathrm{kg}$ & - & - \\
\hline
\end{tabular}




\subsection{Apport en mercure}

Les apports alimentaires en mercure estimés, à partir des quantités d'aliments consommées chez l'individu adulte, sont présentés dans le tableau 5. Les apports en mercure estimés à partir des quantités consommées de la DPH et de l'OMS sont respectivement de $73 \mu \mathrm{g} / \mathrm{semaine}$ et $61 \mu \mathrm{g} / \mathrm{semaine}$. Ils sont au moins 5 fois inférieurs à la Dose Hebdomadaire Tolérable Provisoire (DHTP) préconisée par le comité mixte $\mathrm{FAO} / \mathrm{OMS}(300 \mu \mathrm{g} / \mathrm{semaine})$ qui prend en compte les enfants et les nourrissons. Ces valeurs s'insèrent parmi celles de la littérature qui sont assez disparates. En effet, ces valeurs sont de $70 \mu \mathrm{g} / \mathrm{semaine}$ en France, $217 \mu \mathrm{g} /$ semaine en Allemagne, 5-14 $\mu \mathrm{g} / \mathrm{semaine}$ aux Pays-Bas, 14$21 \mu \mathrm{g} / \mathrm{semaine}$ en Grande Bretagne et $95 \mu \mathrm{g} /$ semaine en Belgique (OMS, 1989; Boisset, 1996).

En considérant un sujet adulte dont la consommation alimentaire totale est constituée uniquement de poissons $(1,08 \mathrm{~kg} / \mathrm{jour}$ selon l'OMS), l'apport estimé serait de $1703 \mu \mathrm{g} /$ semaine; ce qui correspond à 7 fois la valeur de la DHTP. Ainsi, certaines habitudes alimentaires, en particulier chez les populations lagunaires de la Côte d'Ivoire, peuvent exposer certains individus à la toxicité du mercure.

Tableau 5 : apports alimentaires estimés à partir des quantités d'aliments consommées par un individu adulte

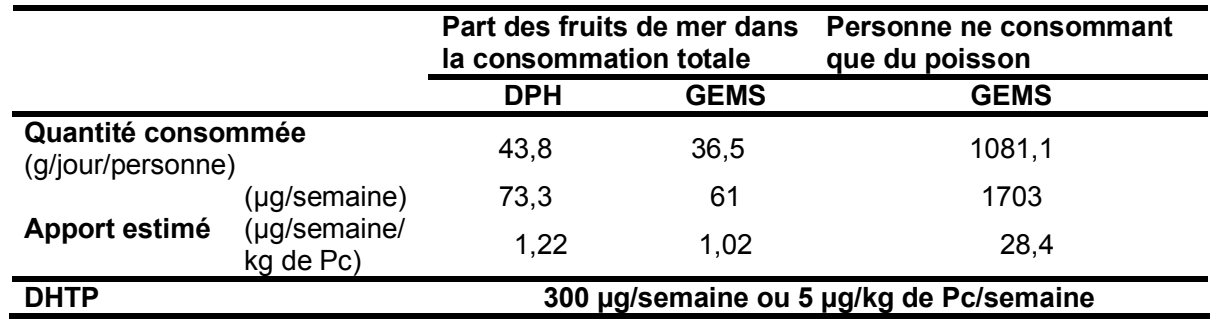

\section{Conclusion}

L'apport en mercure estimé, à partir des concentrations de cet élément toxique et des quantités de poissons consommées quotidiennement est de $73 \mu \mathrm{g} / \mathrm{semaine}$; ce qui représente $25 \%$ de la DHTP. Cette satisfaction générale ne doit pas faire oublier le risque que court une personne qui consomme essentiellement du poisson. En effet, l'apport en mercure ingéré pourrait atteindre $1703 \mu \mathrm{g} /$ semaine chez cette personne; soit 7 fois la DHTP. Par conséquent, il est recommandé aux personnes d'éviter de consommer uniquement les grands prédateurs; au mieux, elles doivent plutôt varier leur alimentation.

\section{Références citées}

AFNOR, 1996. Essais des eaux : protocole d'évaluation d'une méthode alternative d'analyse physico-chimique quantitative par rapport à une analyse de référence. Association Française de Normalisation. Paris, France: XP T ISSN 03353931. $210 \mathrm{pp}$.
Bennett E.G., 1994. Modelling exposure routes of trace metals from sources to man. In : Nriagu J.O. Ed. Changing metal cycles and human health. Berlin, Allemagne: Springer-Verlag. pp 345-456.

Berlin M., 1986. Mercury. In : Friberg L., Nordberg G.F., Vouk V.B. Eds. Handbook on the toxicology of metal. ( $2^{\text {nd }}$ edition), New York, USA: Elsevier. pp 387-445.

Boisset M., 1996. Aspect épidémiologique de l'exposition au mercure et au méthylmercure de la population générale. In : Technique et Documentation Ed. Plomb, cadmium, mercure dans l'alimentation, évaluation du risque. Conseil supérieur d'hygiène publique de France, section de l'alimentation et de la nutrition. Paris, France: Lavoisier. pp 99-112.

Crump K.S., Kjellstrom T., Shipp A.M., Silvers A. \& Stewart A., 1998. Influence of prenatal mercury exposure upon scholastic and psychological test performance: benchmark analysis of a New Zealand cohort. Risk Anal., 18, 701-713.

DPH (Direction des Productions Halieutiques), 2002. Annuaire des statistiques de l'aquaculture 
et des pêches: compile des années 1990 à 2002. Rapport du Ministère de l'Agriculture et des Ressources Animales (MINAGRA), Abidjan, Côte d'Ivoire.

Granjean P., Weihe P., Needham L.L., Burse V.W., Patterson D.G.Jr., Sampson E.J., Jorgensen P.J. \& Vahter M., 1995. Relation of seafood diet to mercury, selenium, arsenic, and polychlorinated bisphenol and other organochlorine concentrations in human milk. Environ. Res., 71, 29-38.

Granjean P., Weihe P., White R.F. \& Debes F., 1998. Cognitive performance of children prenatally exposed to safe levels of methylmercury. Environ. Res., 77, 165-172.

Huss, H.H., 1996. Assurance qualité des produits de mer. Document technique sur les produits de pêche $\mathrm{N}^{\circ} 334$ de la FAO, Rome, Italie.

Métongo B.S. \& Kouamenan K.F., 1991. Concentration en mercure dans les muscles du thon albacore (Thunnus albacares) du golf de Guinée. J. ivoir. Océanol. Limnipl., 1, 1-8.
OMS, 1980. Recommended health based limits in occupational exposure to heavy metals. Technical Report of a WHO study group N ${ }^{\circ} 647$. Geneva, Switzerland.

OMS, 1989. Evaluation de certains additifs alimentaires et contaminants. Rapport Technique du comité mixte FAO/OMS d'experts des additifs alimentaires $N^{\circ} 776$. Genève, Suisse.

OMS, 1996. Lead, cadmium and mercury. In : Health Organization World Ed. Trace elements in human nutrition and health. Geneva, Switzerland. pp 195-216.

OMS, 2003. GEMS/Food regional diets: regional per capita consumption of raw and semi-processed agricultural commodities. Report of Global Environment Monitoring System/Food contamination monitoring and assessment programme. Geneva, Switzerland: Food Safety Department.

Schwarz K., 1977. Essentiality versus toxicity of metals. In : Brown S.S. Ed. Clinical chemistry and chemical toxicology of metals. New York, USA: Elsevier. pp 3-22. 